\title{
EL GOLPE DE ESTADO EN HONDURAS, 2009: UN CASO PARA EL ESTUDIO DE LA MEDIACIÓN INTERNACIONAL
}

\author{
COUP D'ÉTAT IN HONDURAS, 2009: \\ A CASE FOR THE STUDY OF INTERNATIONAL MEDIATION
}

Ronald Sáenz Leandro

María Stephanie Valenciano Hernández

Recibido: 28/02/2019 - Aceptado: 27/05/2019

\begin{abstract}
Resumen
El trabajo examina el proceso de mediación internacional llevado a cabo por el expresidente de Costa Rica, Óscar Arias Sánchez, a raíz de lo acontecido en los días posteriores al golpe de Estado en Honduras, perpetuado el 28 de junio del año 2009. Tiene como objetivo analizar las características del conflicto y enmarcar su intento de gestión como un caso de estudio para la mediación internacional en contextos de paz, lo cual contribuye a discernir sus características y a revisar el papel del mediador dentro de estas coyunturas. Se concluye que la mediación internacional se erige como un área de estudio aún poco desarrollada por la teoría de la resolución alternativa de conflictos (RAC) aplicada en la Ciencia Política, llamando a repensar su papel en la promoción de valores alternativos para el diálogo democrático.
\end{abstract}

Palabras clave: Costa Rica, conflictos de interés público, Honduras, golpe de Estado, mediación internacional, resolución alternativa de conflictos.

\begin{abstract}
The paper seeks to examine the international mediation process carried out by the former president of Costa Rica, Óscar Arias Sánchez, as a result of what happened in the days after the coup d'état in Honduras, perpetuated on June 28 of the year 2009. Its objective is to analyze the characteristics of the conflict and frame its management intent as a case study for international mediation in peace contexts, helping to discern its characteristics and reviewing the role of the mediator within these situations. Concludes that international mediation stands as an area of study little developed by the theory of alternative dispute resolution (ADR) applied in Political Science, calling for rethinking its role for the promotion of alternative values of democratic dialogue.
\end{abstract}

Keywords: Costa Rica, conflicts of public interest, Honduras, coup d'état, international mediation, alternative dispute resolution. 


\section{Introducción}

En el año 2019 se cumple una década del golpe de Estado perpetuado contra el gobierno del expresidente depuesto de Honduras, José Manuel Zelaya Rosales (20062009), el día 28 de junio del año 2009. Este hecho volvió a encender las alarmas de la región en torno a lo que hasta ese entonces se daba por superado en todo el continente: el desplazamiento forzado de un presidente democráticamente elegido, con el apoyo de las Fuerzas Armadas.

De manera natural, el golpe despertó grandes sospechas sobre los logros alcanzados desde la década de los Acuerdos de Paz en Centroamérica, principalmente en lo referente a la pacificación, la desmovilización militar, la democracia electoral y la violación de los Derechos Humanos. En suma, el primer golpe exitoso del siglo XXI latinoamericano ayudó a confirmar los pronósticos de una corriente académica que en los años previos ya venía preocupándose por la eficiencia del gobierno presidencial y la ruptura democrática en este sistema gobierno (Cheibub; Pérez-Liñán; Alcántara, Blondel y Thiébault).

Sobre este tema en particular, debe hacerse notar que la mayoría de estudios académicos centrados en el caso hondureño se han enfocado en dos grandes dimensiones de manera predominante, a saber: la primera centrada en el contexto políticosocial previo y las consecuencias posteriores del golpe (Aguilar; Seligson y Booth; Vásquez; Benítez y Diamint; Mejía y Fernández; Pirker y Núñez; Castro; Rodríguez; Pantanali; Estaun; Treminio, 2015, Treminio, 2016); y una segunda con la mira puesta sobre la reacción de la comunidad internacional y la polarización regional provocada por el conflicto (Bonavitta; Díaz; Gordon y Webber).

En relación con la segunda dimensión -la cual ha sido mucho menos explorada-, este trabajo busca examinar el proceso de mediación internacional llevado a cabo por el expresidente de Costa Rica, Óscar Arias Sánchez, a raíz de lo acontecido en los días posteriores al golpe de Estado en Honduras. Así, se tiene por objetivo caracterizar el conflicto y enmarcar su intento de gestión como un caso de estudio para la mediación internacional en contextos de paz, con lo cual se ayuda a discernir sus características y a revisar el papel del mediador dentro de estas coyunturas.

Para este efecto, se desarrolla un primer apartado dedicado a caracterizar el uso de los métodos de la resolución alternativa de conflictos (RAC) fuera de los contextos organizacionales, enfocándose en la mediación internacional. En segunda instancia se desarrolla un breve esbozo de los sucesos sociopolíticos que enmarcan el golpe de Estado. En lo siguiente, el grueso del trabajo sigue las etapas de la mediación y analiza los pormenores de su implementación. Por último, en la medida en que el presente texto representa apenas una primera aproximación al objeto de estudio, la sección de apuntes finales aporta al discutir los desafíos y limitantes que pueden surgir de un planteamiento como el propuesto, esto de cara a una futura agenda investigación. 
Se parte del entendido de que la acumulación de conocimiento, teorización y experiencia en el estudio de la mediación internacional de conflictos confiere, de entrada, una importancia significativa al tema planteado en el presente trabajo.

\section{Conflictos de interés público y mediación internacional}

A simple vista, el enfoque, la práctica y las respectivas técnicas de la RAC (mediación, negociación y arbitraje) se muestra como un abordaje relegado a las áreas referentes de la gestión empresarial, o bien, de los conflictos organizacionales. No obstante, si se parte de la máxima que afirma que "para que exista política tiene que existir conflicto" (Monedero 78-9), dicha visión reduccionista resulta insuficiente para comprender los conflicto de interés público y sus elementos, en tanto estos pueden llegar a trascender a los individuos o a las organizaciones para manifestarse entre colectividades, convirtiéndose en expresión de "asuntos en disputa [que] afectan [la] propiedad pública o comunitaria o donde están en juego derechos y/o intereses colectivos" (Guillén, Paniagua y Arias 12-13).

Partiendo de esta perspectiva, Hernández propone que los enfoques tradicionales de RAC pueden ser utilizados también como categorías para el análisis político, ya que los acercamientos politológicos a los procesos de mediación, negociación y arbitraje están marcados por el reconocimiento del andamiaje de instituciones establecidas y de los mecanismos formales que asignan las "reglas del juego" en un sistema político determinado. Es decir, reconociendo que un conflicto público en todos los casos está condicionado por influjos ambientales y coyunturales que determinan su gestión.

Para el caso específico de la mediación, Hernández la define como la técnica de RAC que pone en práctica "la intervención de un tercero neutral en un conflicto, con el propósito de ayudar a las partes a resolver sus problemas en un ambiente seguro" (Hernández 114), ya que se parte de que "la persona mediadora mejora el proceso de comunicación ayudando a las partes a definir claramente su problema, a comprender los intereses de cada parte, y a generar opciones para solucionar la disputa" (Hernández 114). Asimismo, se dice que esta persona debe cumplir con el requisito de ser reconocida como legítima, neutral e imparcial para que el proceso de mediación sea exitoso.

En efecto, esta técnica es la mediación de terceros a dos o más partes interactuantes (Pruitt y Kressel) y es, probablemente, una de las formas más antiguas de resolución de conflictos. A lo largo de los años su uso se ha extendido, siendo de importancia en las últimas décadas para la práctica de negociaciones laborales, relaciones internacionales y disputas comunitarias (Lynn y Wall 161).

Dentro de las ventajas del uso de esta técnica se encuentra el principio de disponibilidad según el cual cualquier parte involucrada en la mediación puede terminar el procedimiento sin motivación, lo que se refleja en la ausencia de cualquier 
restricción. Además, alivia a los tribunales de un número significativo de disputas, y sin ser percibido como un juez, el mediador puede encontrar una solución al conflicto que puede satisfacer a ambas partes, y esto sería preferible al hecho de la justicia, según el cual una parte queda insatisfecha (Florea 82).

Ahora bien, es innegable el hecho de que los conflictos pueden trascender su escala nacional y tener consecuencias para los actores más allá de las fronteras de un determinado país. Para Carrascal la mediación internacional forma parte de la práctica habitual de las naciones al menos desde la firma de los Tratados de Westfalia en 1648. Sin embargo, en la actualidad el campo de la mediación internacional ${ }^{1}$ no se encuentra regulado de manera extensa en el seno del sistema de naciones, sino que su marco se encuentra diseminado: la mediación en este campo ha sido ejercida mayormente por organizaciones regionales, organizaciones no gubernamentales (ONG), e incluso líderes o figuras representativas, dotadas de legitimidad por las partes, para llevar a cabo la gestión del conflicto. Tomando esto en cuenta, para el autor, la mediación en la esfera internacional implica:

Un medio diplomático de arreglo de controversias [en el que] a diferencia de los medios jurisdiccionales, los actores que recurren a ella conserva su libertad de acción y de decisión en cuanto a la solución final del conflicto. Además, la solución al mismo se plasma en un acuerdo internacional obligatorio y ésta no tiene que basarse necesariamente en el Derecho Internacional, sino que puede tener en cuenta total o parcialmente elementos de oportunidad politica. Se trata de un instrumento activo: el mediador internacional no se limita a poner en contacto a las partes, sino que también participa, realiza propuestas y negocia (Carrascal 29).

La mediación internacional, como variedad de la técnica convencional, resalta por su carácter multidisciplinar y variopinto en tanto su carácter internacional radica en la presencia de uno o más actores que no representan a una misma nación, en el caso de las partes, o bien por la introducción de la figura del mediador internacional. Estudiar este tipo de variedades en las técnicas tradicionales de RAC permite avanzar en la línea de romper con el molde gerencial que intuitivamente se les ha acuñado.

Estos procedimientos de manera histórica se han aplicado para atender conflictos en contextos de guerra civil o de conflictos internos prolongados (Bercovitch y Rubin; Touval; Sisk; Greig y Diehl; Stipanowich; Vuković). Para los últimos años, este interesante campo se ha extendido hacia América Latina, impulsado sobre todo por la firma de los acuerdos de paz entre el gobierno colombiano y la guerrilla de las Fuerzas Armadas Revolucionarias de Colombia (FARC), apuntalando la figura de Cuba como un país con vocación de mediador (Estrada; Medina; Restrepo).

A pesar de estos esfuerzos, se ha avanzado muy poco en el estudio de las coyunturas de crisis no asociadas -en principio- con el componente bélico. El caso propuesto en este trabajo pretende ofrecer un aporte en esa línea, argumento que un 
proceso de mediación fallido también puede aportar luces para la teoría y la práctica de la resolución alternativa de conflictos que involucra a actores del sistema internacional.

\section{El contexto del golpe de Estado}

José Manuel Zelaya gana las elecciones generales en 2005, asume en enero de 2006 y es el sétimo presidente constitucional de Honduras desde el retorno de la democracia en $1982 .{ }^{2}$ Llegó para asumir la candidatura en representación del Partido Liberal (PLH) de Honduras, resultando electo con un $49.9 \%$ de los votos, frente a un $46.2 \%$ conseguido por Porfirio Lobo, candidato del Partido Nacional (PNH). En el 2006, el presidente del PLH inicia su gestión con una simpatía partidaria cercana al del $25 \%$, muy por debajo de lo reportado en años anteriores, como es el caso del 2001 cuando el Partido Liberal registraba casi un $50 \%$. Este aspecto podía resultar de preocupación, sobre todo para quienes consideran que una fuerte identificación con los partidos políticos es una condición importante para la estabilidad del sistema partidario y la consolidación del sistema democrático (Cruz, Argueta y Seligson 172).

Para el 2008, el barómetro de LAPOP indicaba que la mayoría de los hondureños decía tener niveles medios de confianza en la figura de Zelaya. Así, mientras que los simpatizantes del partido del presidente estaban más inclinados a confiar en la presidencia, los simpatizantes de los partidos de la oposición tendían a perpetuar su desconfianza. Las personas sin una identificación partidaria, sin embargo, reportaban un nivel de confianza al nivel de los partidarios del PLH (Coleman y Argueta 185). Por entonces, Seligson y Booth identificaban a Honduras y a Haití como los países con proporciones más altas de ciudadanos triplemente insatisfechos en el continente (personas antidemocráticas, institucionalmente desleales y con frustración económica). Esto, si bien no predice lo ocurrido en 2009, sí ayuda a explicar algunas de las razones de la fragilidad democrática y la inestabilidad política que alimentaron la crisis desde adentro.

Precisamente un año antes del golpe, el presidente Zelaya sorprende a propios y extraños al realizar un giro inesperado en la política exterior hondureña, gestionando alianza con el entonces presidente de Venezuela, Hugo Chávez. De esta forma, Honduras ingresa en PetroCaribe ${ }^{3}$ y posteriormente, con el apoyo de Chávez, da inicio al proceso de ingreso de Honduras a la ALBA. ${ }^{4}$ Esta situación naturalmente, incentivó una fuerte oposición conformada por las élites económicas, las cúpulas militares-empresariales y los grupos conservadores, quienes consideraban ese accionar contrario a los intereses comerciales del país y las alianzas de larga data con Estados Unidos (Taylor y Ura 115). ${ }^{5}$

Taylor y Ura afirman que estas decisiones tomadas por el entonces presidente Zelaya, fueron objetadas tanto por el Parlamento como por actores políticos de importancia con dominio sobre las principales instituciones del Estado, a saber del Congreso 
Nacional, la Corte Suprema e incluso el Ejército, los cuales consideraban que el presidente se estaba alejando ideológicamente del Partido Liberal (Treminio 153), a su vez que del resto de líderes del Partido Nacional (Castro 53). Al descontento y desgaste de su figura se sumó el intento de influir sobre la elección de los miembros de la Corte Suprema en enero de 2009, y posteriormente en marzo, las publicaciones de algunos medios que interpretaban las declaraciones de Zelaya como adelantos de su deseo de continuar en el poder, por lo que ya se comenzaba a hablar de la posibilidad de una consulta popular (La Prensa párr. 3).

En de marzo de 2009 se anuncia el envío al parlamento de un decreto que proponía establecer una cuarta urna en las papeletas de las elecciones generales de noviembre. El objetivo de dicha urna consistía en preguntar a los ciudadanos hondureños aprobaban o rechazaban la realización de una asamblea constituyente. Para algunas autoridades esta acción fue interpretada como un pretexto para impulsar la reelección presidencial indefinida, prohibida constitucionalmente.

Con el objetivo de dotar de legitimidad dicha acción, posteriormente se anuncia la realización de una encuesta nacional previa no vinculante, la cual determinaría si se colocaba o no la casilla extra en las papeletas de noviembre. Esta decisión, naturalmente, generó críticas internas, pero además activó las alertas de las autoridades internacionales, quienes no dudaron en comparar la estrategia continuista de Zelaya con los casos de reforma electoral llevados a cabo en países como Nicaragua, Colombia, Ecuador, Bolivia y Venezuela (Llanos y Marsteintredet 180-185).

De entre las principales razones para oponerse a la realización de la consulta se encontraba la cuestión de la norma pétrea constitucional, en la donde se incluía el periodo de gobierno y la prohibición absoluta de la reelección presidencial en Honduras. De esta manera, el Tribunal Contencioso Administrativo califica la realización de la consulta como ilegal e inconstitucional, declarándose la nulidad del decreto ejecutivo. Tras este hecho, el parlamento decide aprobar un reglamento para la realización de consultas ciudadanas, estableciendo así un periodo mínimo seis meses, anterior a los procesos electorales, para poder realizar acciones de esta índole (Menjívar 87).

Un punto importante de señalar consiste en que las Fuerzas Armadas, acatando lo estipulado por el Tribunal Contencioso Administrativo, manifiestan la negativa de colaborar en el proceso logístico de realización de la encuesta nacional, lo cual también provoca una serie de despidos y renuncias por parte del cuerpo militar, entre las que destaca la destitución del jefe del Estado Mayor (Benítez y Diamint 149). A pesar de estos hechos, la realización de la consulta continuó en firme, omitiendo con esto los señalamientos de ilegalidad y la crispación política imperante. No obstante, horas antes de iniciar el proceso de consulta, el 28 de junio de 2009 el ejército irrumpe en la casa de habitación del presidente Zelaya para, seguidamente, expatriarlo y colocarlo en un avión con destino a Costa Rica. 
De manera oficial, la justificación legal del golpe se basó en la inconstitucionalidad de la consulta. Sin embargo, como se ha desarrollado, los factores causales y estructurales del mismo trascendían el fundamento legal, apuntando a un Estado en crisis, una gobernabilidad precaria y un modelo de gestión política y económica ineficiente (Barahona 25). ${ }^{6}$

Por su parte, el gobierno de Costa Rica, encabezado por el entonces presidente Óscar Arias Sánchez, cree conveniente iniciar un proceso de mediación para la búsqueda de una solución conveniente a la coyuntura crítica. Es entonces cuando se activa la maquinaria de la política exterior costarricense, generando posicionamientos sucedáneos en la mayoría de los países del continente.

\section{El proceso de mediación}

Antes de comenzar, es importante mencionar que por el carácter -internacional y controversial- de la coyuntura, resulta muy difícil adquirir información de primera mano que explique con detalle cada una de las etapas de la mediación, de ahí el rol estratégico que juegan las memorias para facilitar la labor analítica, (como la publicada en este caso por Bruno Stagno el entonces Ministro de Relaciones Exteriores costarricense). Las memorias políticas brindan al menos una versión de lo sucedido sobre la cual es posible reconstruir muchos de los procesos que se realizan mayormente a puertas cerradas. ${ }^{7}$

La mediación emprendida en este caso puede estudiarse bajo el modelo circular narrativo, inclinado tanto al acuerdo como al mejoramiento de las relaciones entre las partes. Este modelo pretende desplegar la capacidad de intervenir en las partes a través de la información que obtiene sobre la relación y la construcción de nuevas narrativas en las facciones en conflicto, obligando a una intervención activa que dirige el relato de los hechos hacia nuevas historias (Munuera y Garrido 31).

\section{La identificación del conflicto y sus consecuencias}

A partir del apartado anterior es posible comprender la existencia justificada de reservas con respecto a la consulta impulsada por Zelaya, ya que, a pesar del debate en torno a la inconstitucionalidad de la misma, la crispación política llegó embalsar por completo el escenario político hondureño, incluso dentro de las filas del partido de gobierno. De acuerdo con el barómetro LAPOP, un $75.9 \%$ de los hondureños no estaba de acuerdo con dicha consulta y un $70.5 \%$ no consideraba a favor de la formación de una Asamblea Constituyente (Pérez y Argueta 155).

Por otra parte, los datos también indican que más de la mitad de los hondureños no estuvo de acuerdo con la destitución del presidente Zelaya y que más del setenta por ciento se mostraba en abierta discrepancia con su destierro. Además, un $61.1 \%$ consideraba que dicha destitución podía calificarse como golpe de Estado, demostrando con 
esto que, si bien no había un consenso generalizado entre la ciudadanía para apoyar la consulta del presidente, la población no consideraba que la actuación de las Fuerzas Armadas fuera la salida correcta (154-155). Por esto, Stagno afirma que a pesar de que la oposición recorrió un camino previo para inhabilitar legalmente a Zelaya, lo acontecido la mañana de ese domingo 28 de junio le deslegitimó por completo (282).

El Anexo 1 presenta una matriz esquemática con las distintas etapas y posiciones acontecidas en el proceso a partir de este momento.

\section{Preparación de la mediación}

Ante lo sucedido, y luego de una semana de haber sido destituido de su cargo el presidente Zelaya, la balanza de intereses se inclinaba a posicionar al presidente de Costa Rica, Óscar Arias, como quien mediara el conflicto. De acuerdo con Stagno había voces en Honduras que consideraban a Arias la persona idónea para mediar entre las partes, bien porque Costa Rica fue el país que tuvo que recibir al presidente depuesto, o bien porque su discurso y trayectoria le hacían el indicado para algunos (294), especialmente para aquellos actores de la comunidad internacional a los cuales este era políticamente cercano y su figura les servía estratégicamente para incidir sobre el conflicto y posiblemente buscar una salida acorde a sus intereses. ${ }^{8}$

A esto es importante acotar que la propuesta inicialmente no era del agrado de Zelaya, siendo que caía sobre él la presión de los países de la ALBA, para quienes la idea de que Óscar Arias mediara el conflicto no era nada grata, sobre todo si se toman en cuenta las diferencias político ideológicas entre Arias y dicho grupo de países, así como la posible cercanía que tendría con actores del escenario político internacional contrarios a este grupo. ${ }^{9}$ Sin embargo, luego de que el presidente interino de Honduras, Roberto Micheletti, anuncia de forma pública su disposición a aceptar la mediación costarricense, el proceso se encauza con la intervención de la entonces Secretaria de Estado norteamericana, Hillary Clinton (Estaun 295).

Precisamente, la figura de Hillary Clinton juega un papel medular para iniciar el proceso de mediación liderado por Arias. De acuerdo con Stagno, este no sucedió hasta después de una reunión que tuvo el mismo Manuel Zelaya con la entonces Secretaria de Estado, Hillary R. Clinton en Washington, DC (como se detalla en el Anexo). Según narra Stagno, tras un intenso encuentro entre ambos, el presidente depuesto "tuvo que se resignarse a manifestar su anuencia" (295). Este hecho puede tomarse como parte importante para explicar las falencias finales del proceso, siendo que una de las características esenciales de la mediación es la decisión propia de las partes para integrar la resolución del conflicto.

Una vez que la disposición de ambas partes es confirmada, se da inicio a la etapa de acuerdos en torno a la participación y a la escogencia de los equipos de 
negociación. En este punto sucede un primer desacuerdo, pues el equipo de Zelaya hizo pública su petición de contar con Enrique Flores Lanza (su anterior Ministro de la Presidencia) en su delegación, mismo que requería de un salvoconducto para salir de Honduras sin ser detenido por las autoridades. Ante esta petición, el gobierno de Micheletti dio la negativa, poniendo en riesgo la continuidad del proceso. Así, la mediación costarricense, en términos formales y diplomáticos, intentó ser integrativa, sin embargo, no dejó de ser percibida como distributiva por las partes (Estaun 224).

\section{Inicio e interrupción de la mediación}

La mediación acontecida posteriormente mostró sus primeros pasos en la conformación de dos comisiones de representantes, tanto del gobierno de facto, como del grupo de apoyo del presidente depuesto. La intención del presidente Arias era lograr un encuentro cara a cara entre los dos jerarcas, no obstante, una de las partes expresó su negativa, y por lo tanto los encuentros se dieron entre las comisiones de representantes (Stagno 280).

Las rondas de mediación giraron alrededor de siete puntos establecidos por el expresidente Arias luego de las reuniones con las comisiones (311), a saber:

1. La restitución inmediata del Presidente Zelaya.

2. La constitución de un gobierno de unidad nacional.

3. La aprobación de una amnistía.

4. La convocatoria a elecciones generales.

5. La prohibición de la cuarta urna u otra reforma similar.

6. La constitución de una comisión de verificación de los hechos.

7. La reinserción de Honduras en la comunidad internacional.

Cada uno de estos puntos giró en torno a restablecer el orden en Honduras, apegándose a las disposiciones de la Constitución Política, constatándose que ambas partes reclamaban ser defensoras de la misma. Este hecho ofrecía una base sólida para la construcción de un acuerdo. Contrario a lo que se podría apuntar, Stagno acota que ambas delegaciones fueron preparadas con argumentos sólidos, apuntando a su vez a la cantidad de veces previas que ambas partes se habían cruzado en el pasado. Al respecto se hicieron señalamientos que apuntaban al diálogo y a la búsqueda de soluciones para el restablecimiento del orden (306).

Hubo como tal, dos rondas de negociación. En la segunda de estas, las comisiones de representantes discutieron y sellaron los puntos fundamentales de lo que se más tarde se llamó "Acuerdo de San José". Estos fueron previamente aceptados y negociados por ambas partes, empero no les fue entregado de manera oficial hasta el 21 de julio durante una conferencia pública dirigida y convocada por Óscar Arias, en donde se supone que debían cerrar finalmente el proceso de mediación (320). 
No obstante -para sorpresa del mediador y por razones que no quedan del todo claras- al momento del evento público, luego de revisar los alcances del acuerdo, ambas partes declinan la disposición inicial mostrada ante las cámaras. Por un lado, Zelaya señaló el proceso como un fracaso y por el otro, Micheletti lo rechazó alegando que un retorno del presidente depuesto no pasaba solo por una decisión unilateral del ejecutivo sino por un pacto entre instituciones (Stagno 326). Así, Zelaya hizo notar que la vuelta incondicional a la presidencia no quedaba garantizada de manera transparente en el acuerdo, la delegación de Micheletti alegó que el retorno del presidente depuesto no pasaba solo por la decisión unilateral del Ejecutivo, imposibilitándose así el compromiso adquirido previamente. Por esta razón, el proceso se sumergió en un impasse del cual no pudo salir más (Estaun 233).

Zelaya volvería a Honduras en el mes de setiembre, en donde permanecería refugiado en la embajada de Brasil en Tegucigalpa por más de cuatro meses, incluso durante la celebración de los nuevos comicios generales, para los cuales impulsó un boicot pacífico a los mismos que tildó de fraudulentos (Peregil párr. 1).

El 29 de noviembre se realizaron las nuevas elecciones presidenciales en Honduras, resultando ganador Porfirio Lobo, asumiendo la presidencia el 27 de enero de 2010. Este hecho terminó de sepultar la mediación costarricense, siendo que la inercia del cuestionado proceso electoral paulatinamente conllevó al restablecimiento del orden constitucional y a la salida del gobierno de facto. ${ }^{10}$

\section{Apuntes finales}

Las conclusiones que se desprenden de este trabajo pueden dividirse en dos: las que tienen que ver con la aproximación metodológica a al estudio de la mediación internacional, y las que atañen directamente al caso de estudio. Sobre las primeras, al ser el tema específico poco explorado queda de manifiesto que presenta limitantes, sobre todo en lo que tiene que ver con la recopilación de información y la profundización sobre un proceso del cual apenas se llega a conocer escaso material documental, al desarrollarse fundamentalmente en el terreno privado. La principal sugerencia que puede surgir de esta limitante es el planteamiento de entrevistas con actores clave, que permitan tanto ampliar las fuentes como triangular las distintas informaciones, esto con el objetivo de fortalecer la exposición y el adentramiento en la diligencia de la mediación a nivel internacional.

De las segundas, en el trabajo pudo evidenciarse que el desenlace del proceso fue evidentemente infructuoso, esto en tanto careció de un acuerdo final respetado por las partes. No obstante, el "Acuerdo de San José" jugó un papel relevante como referente de negociación para canalizar los ánimos y administrar el tiempo de manera estratégica, hasta el momento de la constitución de un nuevo gobierno en Honduras tras las elecciones. El diálogo fue, por tanto, la principal herramienta puesta 
en práctica, aunado al manejo de la presión internacional focalizada en los medios de comunicación y la diplomacia de Cumbres.

El peso de los factores exógenos al proceso significó todo un desafío para Costa Rica como mediador internacional, ya que su principal carta para gestionar el conflicto era el peso de la imagen del expresidente Arias, mientras que los demás países que intervenían ponían en juego otra serie de recursos para incidir en el proceso, a pesar de que este pudo haber sido la respuesta a intereses internacionales de algunos Estados específicos. El manejo de lo estipulado por los países de la ALBA, por la misma OEA, por Estados Unidos, o incluso Brasil, representó una exigencia de diálogo inmediata.

Como se vio, el peso de dichos factores puede ponderarse dentro los elementos que impidieron la firma de un acuerdo final, ya que la labor de conciliar tantas voces disidentes y de gran peso en América Latina, ciertamente entorpeció el proceso. Sin embargo, también pueden traerse a colación elementos básicos de la mediación que para el caso analizado quedaron en entredicho, a saber: la posible ausencia de voluntariedad de las partes para formar parte proceso y la cuestionable neutralidad de quien dirige el proceso, quien por lapsos pareció mostrarse susceptible hacia determinados intereses políticos, o bien, hacia presiones internacionales de Estados con una mayor injerencia en la agenda internacional.

A pesar de lo anterior, se rescatan los intentos por parte de Costa Rica para propiciar la distancia prudencial de dichos actores, lo cual desde el estudio de la mediación es una estrategia interesante de destacar, en tanto en estos casos es importante que el país mediador mantenga la voz principal dentro de la mediación: si el liderazgo se derrumba se llega a un punto muerto.

A modo de cierre, se ha de decir que la mediación internacional se erige como un área de estudio aún poco desarrollada por la teoría de RAC aplicada en la Ciencia Política. Lo que sucede alrededor de estos procesos es política, y por tanto, merece un abordaje que sopese la buena cantidad de actores que interviene en una determinada correlación de fuerzas. Las dinámicas que ahí acontecen representan la puesta en escena de toda la gama de habilidades y recursos por parte de los países para lograr conseguir sus objetivos, de manera que el presente trabajo pretende haber aportado insumos importantes para la reflexión del caso, pero también para repensar las dinámicas de la RAC, no solo como una herramienta natural del ser humano, sino también como un recurso de poder blando entre las naciones (Nye), esto es, como medio en la promoción de valores alternativos para el diálogo democrático.

\section{Notas}

1 Se parte de la delimitación de mediación internacional planteada por Armas para describir una amplia gama de actividades de terceros, siempre que dicha actividad sea aceptable para los adversarios, y que pretende abatir, resolver o resolver una disputa internacional sin tener que recurrir a la fuerza. 
2 Desde el primer momento, Zelaya se enfrenta con panorama social y económico marcado por una profunda desigualdad social, con tasas altas de analfabetismo, así como de indigencia y pobreza. Asimismo, con una de las economías más pobres y de más bajo crecimiento en América Latina, que colocaban a Honduras en la zona de países en situación de riesgo del continente (Fernández).

Alianza petrolera entre algunos países del Caribe y Venezuela cuyo objetivo reside en la transacción de crudo venezolano en condiciones de pago preferencial.

La Alianza Bolivariana para los Pueblos de Nuestra América-Tratado de Comercio de los Pueblos o ALBA-TCP es una organización internacional de ámbito regional fundada en 2004 y formada por países de América Latina y el Caribe. El ALBA pone énfasis en la lucha contra la pobreza y la exclusión social de acuerdo con doctrinas de izquierda.

Este es un antecedente histórico de suma importancia para comprender el conflicto que se estudia en este caso, en el tanto desde la década de los ochenta se solidificó la relación entre las Fuerzas Armadas Hondureñas y las Estadounidenses, que convirtió a Honduras en un aliado de Estados Unidos para el derrocamiento del gobierno revolucionario de Nicaragua y para evitar el triunfo del FMLN en El Salvador (Benítez y Diamint 147).

En este punto resulta importante recordar brevemente la trayectoria histórica de la institución castrense en Honduras durante el siglo XX. Como apunta Cedeño desde el año 1963 -con el golpe militar al presidente liberal Ramón Villeda- y hasta 1982 -con la promulgación de la nueva Constitución y la reinstauración del gobierno civil-, Honduras estuvo atrapada en un bucle de gobiernos militares. No obstante, a pesar del retorno a los cuarteles, la dinámica de la institución militar no dejó de tener una importante influencia sobre muchos aspectos de la vida política, social y económica en el país, la cual se llegó a acentuar durante los años de la guerra civil en los países vecinos, con el apoyo de la inteligencia militar estadounidense y el apoyo logístico a las fuerzas de contrainsurgencia (213-214). Con el retorno a la democracia en Centroamérica el panorama no llegó a variar abruptamente, perpetuando en este país "la gran dificultad de postular la posibilidad de la existencia de unas fuerzas armadas no deliberantes y subordinadas al poder civil, respetuosas de los derechos políticos y civiles de la población, y con una clara delimitación de su misión en los terrenos de la defensa nacional... la institución castrense ha devenido en una expresión -en sí misma- de nuevos intereses situados por encima de los de esa oligarquía, a la cual -en principio- protegen" (Cedeño 215).

Los autores comprenden los posibles sesgos que esta estrategia pueda tener, sin embargo, es necesario acotar que, hasta la fecha, el presente es el único documento que intenta abordar en forma exhaustiva y exclusiva el proceso de mediación estudiado en este caso. Asimismo, un documento autobiográfico ya ha sido utilizado con fines analíticos en otro estudio para desglosar procesos de aplicación de RAC a nivel internacional, los cuales, por su naturaleza, muchas veces se suelen desarrollar en sesiones privadas. A este respecto, se puede consultar Sáenz.

De acuerdo con los correos revelados de Hillary Clinton, Main señala que el Departamento de Estado en realidad no tenía intención de buscar la resolución de la crisis a través de la OEA -esto por el apoyo que podría recibir ahí Zelaya de algunos estados miembros-, sino a través del proceso de mediación aquí estudiado, el cual se convirtió en su prioridad para atender el asunto.

Es importante recalcar el rol que juegan los países o naciones ajenas al proceso pero que cuentan con un claro interés de influir en el mismo, resultando un factor de riesgo con altas posibilidades de injerencia y modificación del resultado. Para este caso, Nicaragua negó el paso por su territorio de la aeronave que trasladaba al gobierno de facto, mientras que Venezuela 
y Argentina llegaron a emitir declaraciones públicas con miras a descalificar el proceso. A lo anterior se suma la presión constante por parte de Estados Unidos para desarrollar al proceso (Bonavitta). Estas situaciones, con total certeza, modifican el ambiente de la mediación en términos de las posiciones de los participantes.

Si bien el orden se reestablece en términos procedimentales, el proceso electoral que facilitó la transición estuvo teñido de condiciones poco favorecedoras para la transparencia, entre ellas la militarización y el bajo perfil competitivo (Castro 56).

\section{Bibliografía}

Aguilar, Carlos. ¿Cómo y por qué del Golpe de Estado en Honduras? La sinopsis de un proceso de pacificación y democratización fallido en la región. Revista Realidad, 121(2009): 699-707.

Alcántara, Manuel, Jean Blondel y Jean-Louis Thiébault. Presidents and Democracy in Latin America. New York: Routledge, 2018.

Armas, Manuel. La mediación en la resolución de conflictos. Educar 32(2003): 125-136.

Barahona, Marvin. Estado oligárquico, crisis política y corrupción pública. El golpe de estado en Honduras desde una perspectiva de los derechos humanos. Eds. Joaquín Mejía y Víctor Fernández. Tegucigalpa: ERIC-SJ y MADJ, 2010. 23-50.

Benítez, Raúl y Ruth Diamint. La cuestión militar: el golpe de Estado en Honduras como desafío a la democracia y al sistema interamericano. Nueva Sociedad 226(2010): 145-157.

Bercovitch, Jacob y Jeffrey Rubin. Mediation in International Relations: Multiple Approaches to Conflict Management. New York: Palgrave Macmillan, 1992.

Bonavitta, Paola. Golpe de Estado en Honduras: el discurso de Cristina Fernández. Razón y Palabra 74(2011): 1-17.

Carrascal, Ángel. La mediación internacional en el sistema de Naciones Unidas y en la Unión Europea: evolución y retos de futuro. Revista de Mediación 8(2011): 28-33.

Castro, Roque. El golpe en Honduras. Ofensiva conservadora y resistencia. Bajo el volcán 11.17 (2011): 43-74.

Cedeño, Rogelio. Desmovilización militar en América Central: el impacto social y político del estallido de la paz (los años noventa). San José: Distribuidora Editorial Centroamericana de Libros, Servicio de Paz y Justicia en América Latina, 2008.

Coleman, Kenneth y José Argueta. Cultura política de la democracia en Honduras, 2008: el impacto de la gobernabilidad. San Salvador: IUDOP, 2008.

Cruz, José Miguel, José Argueta y Mitchell Seligson. Cultura política de la democracia en Honduras, 2006. San Salvador: IUDOP, 2007.

Cheibub, José Antonio. Presidentialism, Parliamentarism, and Democracy. Cambridge: Cambridge University Press, 2007.

Díaz, Silvana. La actuación de la OEA frente al golpe de Estado de Honduras (2009). Tesina para obtener el título de especialización en Relaciones Internacionales y Seguridad en América Latina. Facultad Latinoamericana de Ciencias Sociales, Sede Ecuador, 2013.

Estaun, Eva. Causas y consecuencias de una crisis politica: fragilidad institucional. Impunidad. Honduras junio 2009-enero 2014. Tesis doctoral. Universidad Nacional de Educación a Distancia, Instituto Universitario General Gutiérrez Mellado, Madrid, 2015. 
Estrada, Jairo. "Elementos para una problematización básica del Punto 3 "Fin del conflicto" y del Punto 6 "Implementación, Verificación y Refrendación" de la Agenda de diálogos de La Habana”. Paz en Colombia: perspectivas, desafíos, opciones. Eds. Sara Victoria, Eduardo Rueda, y Pablo Gentili. Buenos Aires: CLACSO, 2016. 91-108.

Fernández, Recaredo. Bipartidismo, pobreza y golpe de Estado. El golpe de Estado en Honduras desde una perspectiva de los derechos humanos. Eds. Joaquín Mejía y Víctor Fernández. Tegucigalpa: ERIC-SJ y MADJ, 2010. 160-196.

Florea, Bogdan. Mediation as an efficient way of saving public money. The Juridical Current, 72(2018): 78-87.

Gordon, Todd y Jeffery R. Webber. Canadian Geopolitics in Post-Coup Honduras. Critical Sociology 40.4(2014): 601-620.

Greig, J. Michael y Paul Diehl. International Mediation. Cambridge: Polity Press, 2012.

Guillén, Sergio, Francisco Paniagua y Randall Arias. Manual de formación en negociación basada en intereses. San José: Fundación para la Paz y la Democracia, 2011.

Hernández, Evelyn. “Un estudio desde la ciencia política de la mediación en Centroamérica: el caso de Costa Rica". Politica y Sociedad 50.1 (2013): 113-144.

La Prensa. Mel acepta que desea seguir en el poder. La Prensa Mar, 2009.

Lynn, Ann y James Wall. Mediation: A Current Review. The Journal of Conflict Resolution 37.1 (1993): 160-194.

Llanos, Mariana y Leiv Marsteintredet. Ruptura y continuidad: la caída de "Mel" Zelaya en perspectiva comparativa. América Latina Hoy, 55(2010): 173-197.

Main, Alexander. Hillary Clinton's Emails and the Honduras Coup. Center for Economic and Policy Research Sep, 2015.

Medina, Medófilo. El proceso de paz de la Habana en la historia y en la actualidad. Paz en Colombia: perspectivas, desafíos, opciones. Eds. Sara Victoria, Eduardo Rueda y Pablo Gentili. Buenos Aires: CLACSO, 2016. 73-82.

Mejía, Joaquín y Víctor Fernández. El golpe de Estado en Honduras desde una perspectiva de los Derechos Humanos. Tegucigalpa: ERIC-SJ y MADJ, 2010.

Menjívar, Omar. El golpe de Estado desde una perspectiva jurídica. El golpe de Estado en Honduras desde una perspectiva de los derechos humanos. Eds. Joaquín Mejía y Víctor Fernández. Tegucigalpa: ERIC-SJ y MADJ, 2010. 85-114.

Monedero, Juan Carlos. El gobierno de las palabras: política para tiempos de confusión. Madrid: Fondo de Cultura Económica, 2011.

Munuera, Pilar y Salvador Garrido. Innovación en mediación a través de la intervención narrativa: desmitificando el principio de neutralidad. Revista de Mediación, 8(2015): 25-35.

Nye, Joseph. Soft Power: The Means to Success in World Politics. New York: Public Affairs, 2005.

Pantanali, Antonella. Crisis y golpe de Estado en Honduras. XI Congreso Nacional y IV Congreso Internacional sobre Democracia. Rosario, Santa Fe, Sep. 8-11, 2014. Rosario: Universidad Nacional de Rosario, Consejo de Estudios Interdisciplinarios Económicos y Políticos, 2014. 1-15.

Peregil, Francisco. Porfirio Lobo gana las elecciones de Honduras. El País, 2009.

Pérez, Orlando y José Argueta. Cultura política de la democracia en Honduras, 2010: consolidación democrática en las Américas en tiempos difíciles. San Salvador: IUDOP, 2010.

Pérez-Liñán, Anibal. Presidential Impeachment and the New Political Instability in Latin America. Cambridge: Cambridge University Press, 2007.

Pirker, Kristina y Omar Núñez. Cuatro hipótesis y un corolario en torno al golpe de Estado en Honduras. OSAL 28(2010): 119-143. 
Pruitt, Dean G y Kenneth Kressel. Introduction: An overview of mediation research. Mediation research: the process and effectiveness of third-party intervention. Eds. Kenneth Kressel y Dean. G. Pruitt. San Francisco, CA: Jossey-Bass, 1989. 1-27.

Restrepo, Javier. Colombia: Nota sobre los borradores de La Habana hasta ahora. Paz en Colombia: perspectivas, desafíos, opciones. Eds. Sara Victoria, Eduardo Rueda, y Pablo Gentili. Buenos Aires: CLACSO, 2016. 83-88.

Rodríguez, Cecilia. Volver a empezar: análisis de las elecciones hondureñas tras el golpe de Estado. América Latina: política y elecciones del bicentenario (2009-2010). Eds. Manuel Alcántara y María Laura Tagina. Madrid: Centro de Estudios Políticos y Constitucionales, 2011. 213-239.

Sáenz, Ronald. Esbozo para interpretar un proceso de negociación internacional: el establecimiento de relaciones diplomáticas entre Costa Rica y la República Popular China. Revista Análisis de la Realidad Nacional 118(2017): 77-105.

Seligson, Mitchell y John Booth. Predicting Coups? Democratic Vulnerabilities, The Americas Barometer and the 2009 Honduran Crisis. Americas Barometer Insights 1(2009): 1-6.

Sisk, Timothy. International Mediation in Civil Wars. New York: Routledge, 2009.

Stagno, Bruno. Los caminos menos transitados: la administración Arias Sánchez y la redefinición de la política exterior de Costa Rica, 2006-2010. Heredia: EUNA, 2013.

Stipanowich, Thomas J. The International Evolution of Mediation: A Call for Dialogue and Deliberation. Victoria University of Wellington Law Review, 46(2015): 1191-1243.

Taylor, Michelle y Joseph Ura. Public Opinion and Conflict in the Separation of Powers: Understanding the Honduran Coup of 2009. Journal of Theoretical Politics 25.1(2012): 105-127.

Touval, Saadia. Mediating the Yugoslav Wars: The Critical Years 1990-95. New York: Palgrave, 2002.

Treminio, Ilka. Las reformas a la reelección presidencial del nuevo siglo en América Central: tres intentos de reforma y un golpe de Estado. Política y gobierno 22(2015): 147-217.

Treminio, Ilka. ¿Cómo borrar la letra escrita en piedra? Norma pétrea y reelección presidencial en Honduras. Anuario de Estudios Centroamericanos 42(2016): 237-260.

Vásquez, Guillermo. Crisis política en Honduras: ¿adolescencia o madurez democrática? Revista Electrónica Iberoamericana, 3.2(2009): 29-62.

Vuković, Siniša. International Multiparty Mediation and Conflict Management: Challenges of Cooperation and Coordination. New York: Routledge, 2016.

Ronald Sáenz Leandro. Costarricense. Licenciado en Ciencias Políticas por la Universidad de Costa Rica. Investigador de FLACSO-Sede académica Costa Rica.

Contacto: ronald.saenz@ucr.ac.cr

ORCID: 0000-0001-8717-1870

María Stephanie Valenciano Hernández. Costarricense. Egresada de la Licenciatura en Ciencias Políticas de la Universidad de Costa Rica. Becaria actual en el Instituto de Investigaciones Sociales (IIS-UCR).

Contacto: maría.valenciano_h@ucr.ac.cr

ORCID: 0000-0001-6402-6961 


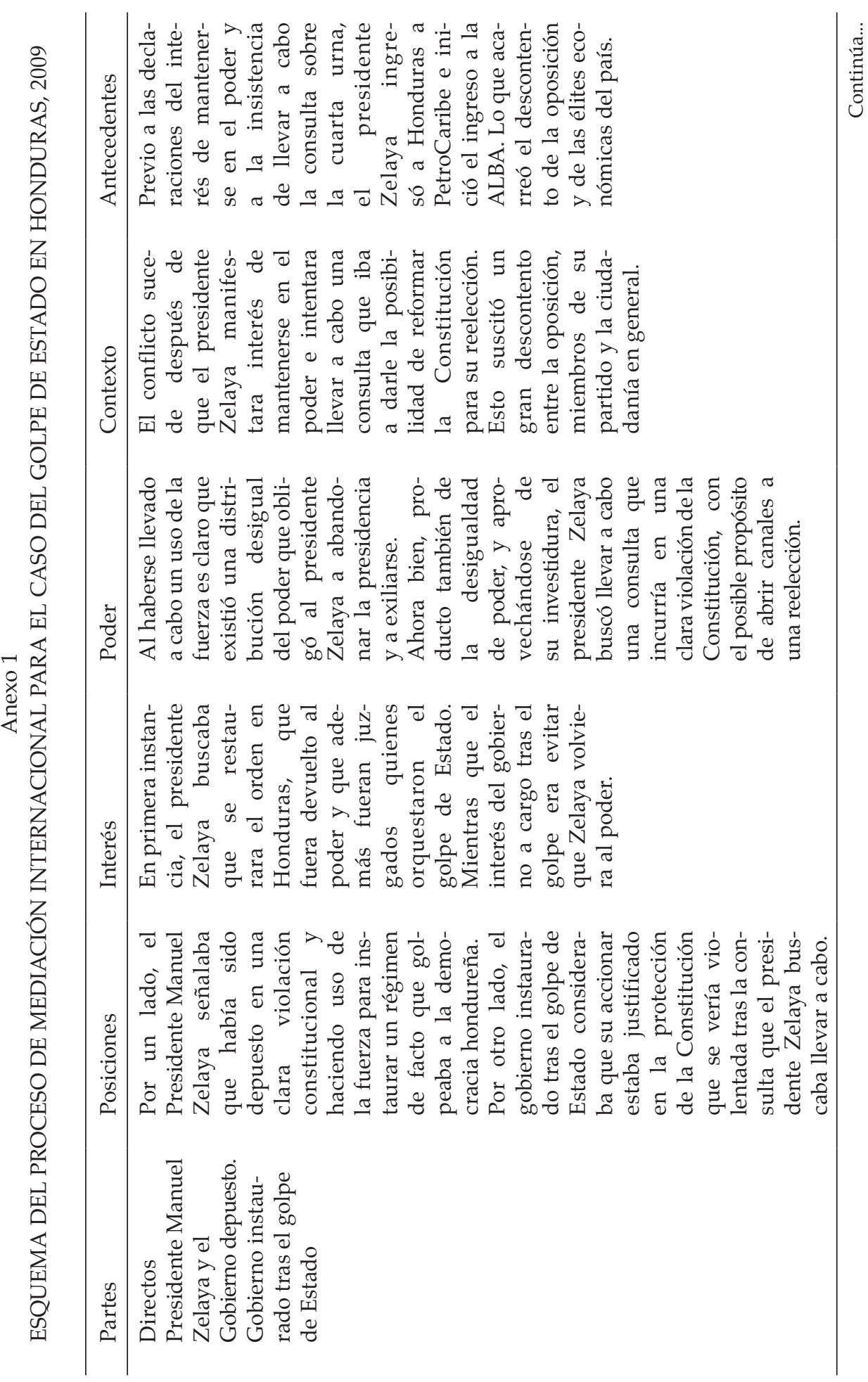




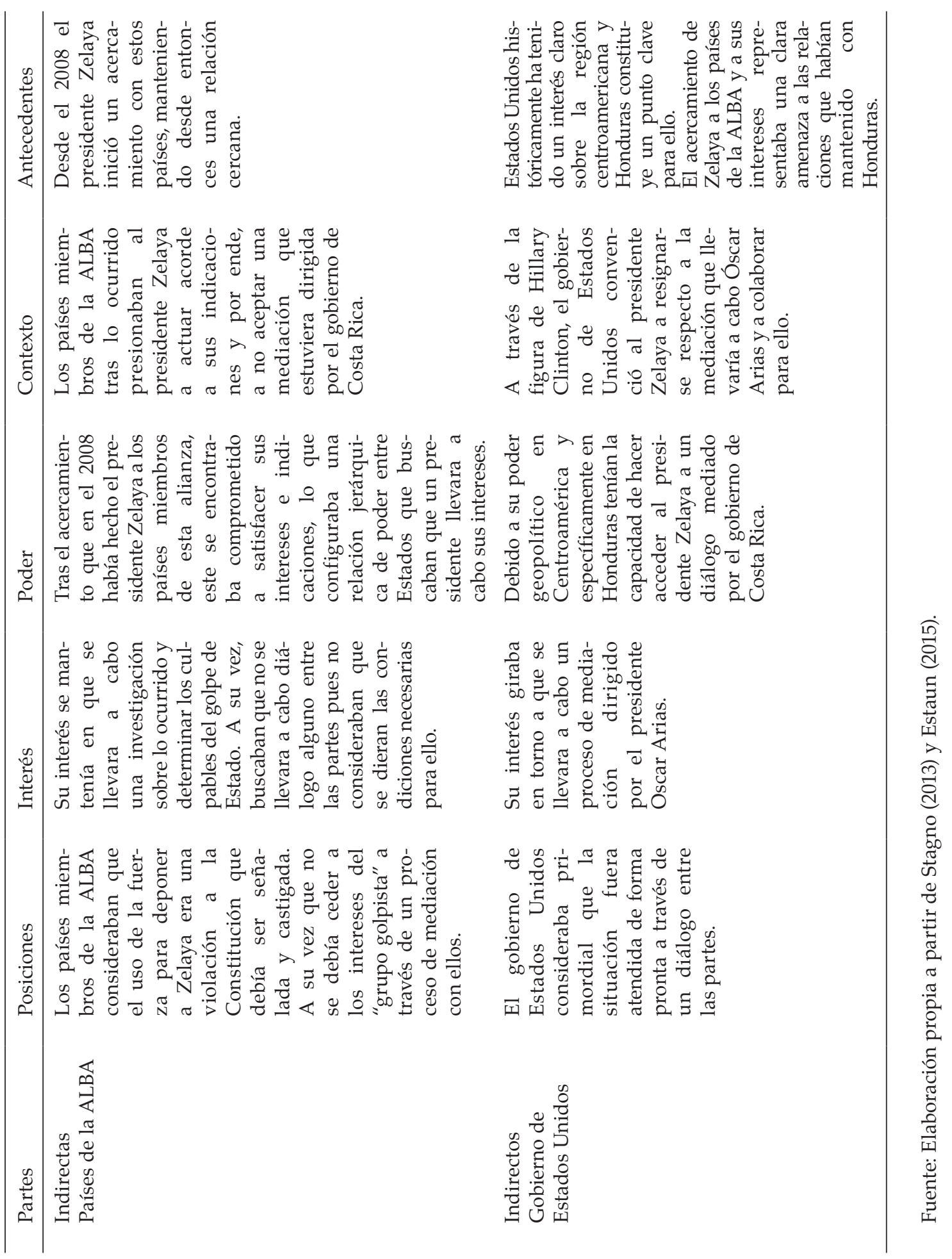


